\title{
A Parallel Algorithm for the Technology Mapping of LUT-Based FPGAs
}

\author{
Vamsi Boppana ${ }^{1 \star}$ and Prashant Saxena $a^{2 \star \star}$ and Prithviraj Banerjee ${ }^{1 \star}$ and \\ W. Kent Fuchs ${ }^{1 \star}$ and C. L. Liu ${ }^{2 \star \star}$ \\ 1 Coordinated Science Lab, 1308 W. Main St. \\ 2 Department of Computer Science, Digital Computer Lab, 1304 W. Springfield Ave. \\ University of Illinois at Urbana-Champaign, Urbana, IL 61801, USA.
}

\begin{abstract}
Flowmap ([1]) was the first delay-optimal algorithm for the technology mapping of LUT-based FPGAs. However, even though this algorithm is polynomial, rapid prototyping using FPGAs requires faster solutions. This paper provides an efficient parallelization of FLOWMAP that minimizes locking on shared memory architectures. The influence of scheduling strategies and technology-specific parameters on speedups is studied. The expected running time is also analyzed. The parallel algorithms yield speedups of around 4 to 5 on 8 processors.
\end{abstract}

\section{Introduction}

Parallel processing has extensive applications in all phases of VLSI design ([2]). A key step in the design cycle that does not yet have a parallel solution is the field programmable gate array (FPGA) technology mapping problem. Fast technology mapping is vital in maintaining one of the main advantages offered by FPGAs, viz., a small design turn-around time for rapid, interactive prototyping.

An LUT-based FPGA consists of an array of programmable logic blocks containing lookup tables (LUTs). A K-input LUT can realize any arbitrary boolean function of upto $K$ variables. The process of realizing a general boolean network using LUTs is called FPGA technology mapping. Cong \& Ding ([1]) developed a polynomial algorithm, FlowMAP, for optimal-delay technology mapping for LUT-based FPGAs under the unit delay model. FlowmaP yields minimum depth FPGA realizations for combinational circuits. It first computes labels for all the nodes in the circuit in topological order. A node's label represents the depth of the optimal realization of its fanin cone. The second phase uses the labels to select gates to be realized by each LUT. Since the second phase runs in linear time, most of the run-time is spent in the labeling phase.

FLOWMAP is an efficient polynomial algorithm; therefore, sequential algorithmic advances are unlikely to yield significantly faster solutions to the problem. We provide an alternative by exploiting the parallelism available in the labeling phase. Our parallel algorithms are designed for shared memory architectures and guarantee a final mapping identical to the corresponding sequential solutions.

* Supported in part by the Semiconductor Research Corporation and the Joint Services Electronics Program under grants 95-DP-109 and N00014-96-1-0129 respectively.

** Supported in part by the National Science Foundation under grant MIP-9222408. 


\section{Parallelization of FLOWMAP}

The computation of a node label involves solving a flow problem on a network that depends only on the labels in the fanin cone of the node. A simple parallel strategy to compute node labels uses a centralized task queue. This event-driven scheme starts with the queue containing only the primary inputs (PIs), and places a node into the queue as soon as the labels of all its fanins are computed. However, this requires excessive synchronization. First, whenever the label computation of a node is completed, each of the fanouts of that node must be locked (one at a time) so that their counts of remaining fanin computations may be decremented and any node with count 0 may be placed in the task queue. Second, the task queue is a hot spot, with every idle processor competing for nodes in it.

In contrast, our approach works on the levelized circuit graph; level $i$ containing all nodes whose maximum distance from any PI is $i$. Computation of the labels proceeds level by level, starting with level 0 (the PIs). Correct label computation across levels can be ensured by a barrier at the end of each level. The scheduling of nodes within a level may be static or dynamic. In the case of static scheduling, no locking is required, since the only time a node's label is written is when the level containing the node is being processed. But this node cannot be in any fanin cone currently being processed. Therefore, it is safe to read and write its label without locking. With dynamic scheduling, although labels can still be written without any locking, we need to lock the variable pointing to the next available node. However, our experiments show that this overhead is not significant. The memory requirements for the level-based schemes are small. The dynamic memory required by a processor is bounded by the size of a fanin cone. The static memory requirements are comparable to those of the sequential algorithm because the circuit data structure is read-only.

A potential disadvantage of our approach is the possibility of processors being idie even when work is available in subsequent levels. However, the probability of this occurring becomes small with sufficiently large circuits. Circuits for which this parallelization would be most beneficial, would have near-uniform level sizes with a large number of nodes at each level, and similar-sized fanin cones within each level. In general, speedups on non-ideal circuit topologies degrade less with dynamic scheduling than with static scheduling.

Our implementation of the level-based scheme uses an array levellist $[i][1$.. levelsize [i]] for the levelsize[i] nodes in the graph at level $i$. The pseudocode for static-interleaved scheduling, shown in Figure 1, uses no locking. The pseudocode for dynamic scheduling (Figure 2) requires a lock on the pointer to the next available node. However, we do not need to lock the global label and cut_nodes arrays that store the optimal labels and cuts.

\section{Analysis of Expected Running Time}

Let $n, m$ and $p$ respectively represent the total number of nodes and edges in the circuit graph and the number of processors in use. Let $L_{0}, L_{1}, \ldots, L_{\nu_{l}}$ denote the levels in the levelized graph, with $s_{i}$ being the number of nodes in 
procedure STATIC-FLOWMAP

$j \leftarrow$ my_processor_ID;

while $(j \leq$ levelsize $[i])$

curr_node $\leftarrow$ levellist $[i][j]$

compute the label and optimum cut for node curr_node;

write this label to label[curr_node] and the cut to cut_nodes/curr_node];

$j \leftarrow j+$ num_processors;

Fig. 1. Pseudocode for parallel label computation using static-interleaved scheduling

\section{procedure DYNAMIC-FLOWMAP}

/* Start with curr_node $\leftarrow 0$ on each processor, and the global next_node $\leftarrow 1 *$ /

while (curr_node $\geq 0$ )

lock next_node;

if (next_node $\leq$ levelsize[i]) then

curr_node $\leftarrow$ levellist[i][next_node];

next_node $\leftarrow$ next_node +1 ;

else curr_node $\leftarrow-1$

unlock next_node;

if (curr_node $\geq 0$ )

compute the label and optimum cut for node curr_node; update label[curr_node] and cut_nodes[curr_node];

Fig. 2. Pseudocode for parallel label computation iterations using dynamic scheduling

$L_{i}$. If $t_{i}^{\max }$ is the maximum of the label computation times for the nodes in $L_{i}$, the barrier synchronization implies that the total label computation time for the graph is at most $\sum_{i=0}^{\nu_{l}}\left(t_{i}^{\max } .\left\lceil s_{i} / p\right\rceil\right)$ in the static-interleaved case. With dynamic scheduling, each level $L_{i}$ also requires $s_{i}$ lock-unlock operation pairs that cannot be pairwise concurrent. If the average time for one such pair is $t_{l}$, the label computation time increases by $n t_{l}$ units. However, the dynamic balancing reduces the $\sum_{i=0}^{\nu_{l}}\left(t_{i}^{\max } .\left\lceil s_{i} / p\right\rceil\right)$ term to $\sum_{i=0}^{\nu_{l}}\left(t_{i}^{a v g} .\left\lceil s_{i} / p\right\rceil\right)$.

The initial topological sorting and level computation uses $3 m$ time units. Another $m$ units are required for the mapping phase. Let $C$ and $c^{\prime}$ be the times required to respectively create and reactivate a thread (typically, $c^{\prime} \ll C$ ). Then, the total overhead associated with thread management is $p\left(C+\nu_{l} c^{\prime}\right)$.

Thus, the run-times for the static-interleaved and dynamic schemes are given by $4 m+p\left(C+\nu_{l} c^{\prime}\right)+\sum_{i=0}^{\nu_{l}}\left(t_{i}^{\max } .\left\lceil s_{i} / p\right\rceil\right)$ and $4 m+p\left(C+\nu_{l} c^{\prime}\right)+\sum_{i=0}^{\nu_{1}}\left(t_{i}^{a v g} .\left\lceil s_{i} / p\right\rceil\right)+$ $n t_{l}$ respectively. In contrast, the sequential run-time is $3 m+\sum_{i=0}^{\nu_{l}} s_{i} t_{i}^{\text {avg }}$, since the $m$ units required for level computation and the $p\left(C+\nu_{l} c^{\prime}\right)$ units required for thread management in the parallel algorithms are not needed here.

\section{Experimental Results}

We tested our parallel implementations of FLOWMAP on several large circuits from the MCNC ' 91 CML suite on a SGI Power Challenge multiprocessor. All circuits were first decomposed into a 2-input network of simple gates. This was followed by the actual technology mapping on 2, 4, 6 and 8 processors under both static-interleaved and dynamic scheduling policies.

Table 1 presents the run-times and speedup figures obtained with $K=5$ 
( 5 being typical for many industrial FPGAs). The speedups obtained for the smaller circuits indicate that the parallelism inherent in them is limited by the small number of gates in each level. However, larger circuits, towards which the parallelization is targeted, yield substantially better speedups and scalability. Dynamic scheduling results in slightly better scalability despite the overhead of the task queue. Running times are similar under static-interleaved and dynamic schedules. This implies that the overhead involved in the task queue synchronization is quite small, and that the times required for the processing of different nodes within a level are comparable.

Table 1. Sequential run-times in seconds and parallel speedups (with $K=5$ )

\begin{tabular}{|c|c|c|c|c|c|c|c|c|c|c|}
\hline \multirow{3}{*}{ Circuit } & \multirow{3}{*}{$\begin{array}{c}\text { Optima } \\
\text { depth }\end{array}$} & \multirow{3}{*}{$\begin{array}{l}\text { Sequential } \\
\text { run-time }\end{array}$} & \multicolumn{4}{|c|}{ Static speedup } & \multicolumn{4}{|c|}{ Dynamic speedup } \\
\hline & & & \multicolumn{4}{|c|}{ \# processors } & \multicolumn{4}{|c|}{ \# processors } \\
\hline & & & $\overline{2}$ & 4 & 6 & 8 & 2 & 4 & 6 & 8 \\
\hline $\operatorname{apex} 7$ & 4 & 12.43 & 1.4 & 1.9 & 2.4 & 2.5 & 1.4 & 1.9 & 2.4 & 2.1 \\
\hline $\mathrm{i} 7$ & 2 & 57.87 & 1.5 & 2.1 & 2.6 & 2.9 & 1.4 & 2.1 & 1.9 & 3.3 \\
\hline apex6 & 5 & 10.97 & 1.2 & 2.3 & 3.3 & 3.9 & 1.2 & 2.4 & 3.3 & 3.7 \\
\hline $\mathrm{c} 2670$ & 7 & 89.26 & 1.6 & 2.6 & 3.3 & 4.3 & 1.7 & 2.9 & $\overline{3.6}$ & $\overline{4.7}$ \\
\hline pair & 5 & 50.05 & 1.6 & 2.6 & 3.5 & 3.9 & 1.5 & 2.8 & 3.5 & 4.1 \\
\hline c5315 & 8 & 276.84 & 1.5 & 2.3 & 3.1 & 4.1 & 1.4 & 2.1 & 2.7 & 3.3 \\
\hline i10 & 13 & 389.34 & 1.4 & 2.3 & 3.5 & 4.0 & 1.5 & 2.4 & 3.5 & 4.1 \\
\hline
\end{tabular}

We also studied the speedups obtained with different values of $K$. Increasing the value of $K$ increases the grain size of the available parallelism, since each network flow sub-problem is solved in $O(K \mathrm{~K})$ time. However, this effect is often offset by the increased imbalance created in small-sized levels due to the larger grain size. Thus, the speedups obtained depend primarily on the circuit topology. As an illustration, although $K=7$ resulted in the best speedup for i7 (6.9 on 8 processors), it was the worst choice for $\mathrm{c} 5315$ ( 2.8 on 8 processors).

\section{Conclusions}

In this paper, we presented a strategy for the parallelization of FLOWMAP that yields good speedups and scalability by addressing grain size and load balance.

FloWMAP has been extended to the general delay model by Yang \& Wong ([3]) by generalizing the labeling phase. Our parallel approach can also be extended to their algorithm in a manner analogous to that described in this paper.

\section{References}

1. Cong, J., and Y. Ding, "FlowMap: An Optimal Technology Mapping Algorithm for Delay Optimization in Lookup-Table Based FPGA Designs," IEEE Trans. Computer-aided Design 13 (1), January 1994, 1-13.

2. Banerjee, P., Parallel Algorithms for VLSI Computer-Aided Design, Prentice Hall, Inc., Englewood Cliffs, NJ, 1995.

3. Yang, H., and D. F. Wong, "Edge-Map: Optimal Performance Driven Technology Mapping for Iterative LUT Based FPGA Designs," Digest IEEE Intl. Conf. on $C A D$, November 1994, 150-155. 